\title{
Cost Effectiveness of Blinatumomab Versus Inotuzumab Ozogamicin in Adult Patients with Relapsed or Refractory B-Cell Precursor Acute Lymphoblastic Leukemia in the United States
}

\author{
Thomas E. Delea ${ }^{1}$ (1) $\cdot$ Xinke Zhang $^{2} \cdot$ Jordan Amdahl' $^{1}$ - Diana Boyko ${ }^{1} \cdot$ Franziska Dirnberger $^{3} \cdot$ Marco Campioni $^{3}$. \\ Ze Cong ${ }^{2}$
}

Published online: 20 June 2019

(c) The Author(s) 2019

\begin{abstract}
Background and Objective The TOWER and INO-VATE-ALL trials demonstrated the efficacy and safety of blinatumomab and inotuzumab ozogamicin (inotuzumab), respectively, versus standard-of-care (SOC) chemotherapy in adults with relapsed or refractory (R/R) B-cell precursor acute lymphoblastic leukemia (ALL). The cost effectiveness of blinatumomab versus inotuzumab has not previously been examined.

Methods Cost effectiveness of blinatumomab versus inotuzumab in R/R B-cell precursor ALL patients with one or no prior salvage therapy from a United States (US) payer perspective was estimated using a partitioned survival model. Health outcomes were estimated based on published aggregate data from INO-VATE-ALL and individual patient data from TOWER weighted to match patients in INO-VATE-ALL using matching adjusted indirect comparison (MAIC). Analyses were conducted using five approaches relating to use of anchored versus unanchored comparisons of health outcomes and, for the anchored comparisons, the reference treatment to which treatment effects on health outcomes were applied. Estimates from TOWER including the probabilities of complete remission and allogeneic stem-cell transplant (allo-SCT), overall and eventfree survival, utilities, duration of therapy, and use of subsequent therapies were MAIC adjusted to match INO-VATE-ALL. Costs of treatment, adverse events, allo-SCT, subsequent therapies, and terminal care were from published sources. A 50-year time horizon and $3 \%$ annual discount rate were used.

Results Incremental costs for blinatumomab versus inotuzumab ranged from US $\$ 7023$ to US\$36,244, depending on the approach used for estimating relative effectiveness. Incremental quality-adjusted life-years (QALYs) ranged from 0.54 to 1.78. Cost effectiveness for blinatumomab versus inotuzumab ranged from US $\$ 4006$ to US $\$ 20,737$ per QALY gained. Conclusions Blinatumomab is estimated to be cost effective versus inotuzumab in R/R B-cell precursor ALL adults who have received one or no prior salvage therapy from a US payer perspective.
\end{abstract}

Electronic supplementary material The online version of this article (https://doi.org/10.1007/s40273-019-00812-6) contains supplementary material, which is available to authorized users.

\section{Thomas E. Delea}

tdelea@pai2.com

1 Policy Analysis Inc. (PAI), 4 Davis Court, Brookline, MA 02445, USA

2 Amgen Inc., Thousand Oaks, CA, USA

3 Amgen (Europe) GmbH, Zug, Switzerland

\section{Introduction}

Acute lymphoblastic leukemia (ALL) is a rare and fatal hematologic malignancy characterized by the overproduction of immature lymphocytes (lymphoblasts) that replace healthy lymphocytes, leading to neutropenia, anemia, and thrombocytopenia [1]. ALL affects fewer than ten per million adults annually in the United States (US) [2]. Adults with relapsed or refractory (R/R) B-cell precursor ALL have a poor prognosis and historically have had few treatment options, with most receiving standard of care (SOC) salvage chemotherapy [3, 4]. Studies of patients with relapsed ALL receiving SOC chemotherapy have reported median overall 


\section{Key Points for Decision Makers}

The TOWER and INO-VATE-ALL trials demonstrated the efficacy and safety of blinatumomab and inotuzumab ozogamicin (inotuzumab), respectively, in relapsed or refractory (R/R) B-cell precursor acute lymphoblastic leukemia (ALL).

This study used a partitioned survival model and five different approaches for estimating relative effectiveness using results from a matching adjusted indirect comparison (MAIC) of TOWER and INO-VATE-ALL trials to compare the cost effectiveness of blinatumomab versus inotuzumab in patients with R/R B-cell precursor ALL who have received one or no prior salvage therapy from a United States (US) healthcare payer perspective.

Regardless of the approach employed, blinatumomab was cost effective versus inotuzumab, with incremental cost-effectiveness ratios (ICERs) ranging from US $\$ 4006$ to US\$20,737 per quality-adjusted life-year gained, which are below commonly accepted ICER thresholds in the US.

A variety of different approaches for estimating relative effectiveness based on indirect comparisons may be employed in economic evaluations, which may have an important impact on model results and conclusions. Investigators should provide a rationale for the specific approach that they employ and examine the impact of alternative approaches in sensitivity analyses.

survival (OS) of 4.5-6 months and a 5-year OS of $<10 \%$ $[5,6]$.

Blinatumomab is a bispecific monoclonal antibody construct that enables CD3-positive $\mathrm{T}$ cells to recognize and eliminate CD19-positive ALL lymphoblasts [7]. It is approved by the US Food and Drug Administration (FDA) for the treatment of R/R B-cell precursor ALL $[8,9]$. The efficacy and safety of blinatumomab in adult patients with Philadelphia chromosome ( $\mathrm{Ph}$ )-negative R/R B-cell precursor ALL was examined in the TOWER study, a phase III, multicenter, international, randomized, open-label trial in which a total of 405 patients were assigned to receive blinatumomab $(N=271)$ or SOC chemotherapy $(N=134)$ (NCT02013167) [7]. Patients receiving blinatumomab had improved OS compared with those receiving SOC (median 7.7 vs 4.0 months, respectively; hazard ratio [HR] 0.71; 95\% confidence interval [CI] 0.55-0.93; $p=0.01$ ) [7]. The proportion of patients with complete remission with full hematological recovery (CR) within 12 weeks of treatment initiation was higher in patients receiving blinatumomab versus those receiving SOC (34\% vs $16 \%$, respectively; $p<0.001$ ) [7]. Health-related quality of life was also improved in patients receiving blinatumomab versus those receiving SOC [10]. A total of $24 \%$ of the patients in each treatment group underwent allogenic stem cell transplant (allo-SCT). Grade 3 or higher cytokine release syndrome (CRS) was observed in $5 \%$ of patients receiving blinatumomab and no patients receiving SOC [7].

Inotuzumab ozogamicin (inotuzumab) is a humanized anti-CD22 monoclonal antibody conjugated to calicheamicin, a cytotoxic antibiotic agent [11]. Inotuzumab targets and binds to the $\mathrm{CD} 22$ protein, found on the surface of ALL cells, and releases calicheamicin, leading to cell death [12]. Inotuzumab is approved in the US for adult patients with R/R B-cell precursor ALL based on results of INO-VATEALL, a phase III, multicenter, international, randomized, open-label trial (NCT01564784) [13, 14]. Eligible patients in INO-VATE-ALL $(N=326)$ were adults with either $\mathrm{Ph}-$ or $\mathrm{Ph}+\mathrm{R} / \mathrm{R}$ B-cell precursor ALL with $\geq 5 \%$ bone marrow blasts who had received one or no prior salvage chemotherapy regimen for ALL. The CR rate was higher among patients receiving inotuzumab than SOC (34\% vs $16 \% p=0.0001$ ) [15]. In long-term follow-up, median OS was 7.7 versus 6.2 months with inotuzumab versus SOC (HR 0.75; 97.5\% CI 0.568-0.993; $p=0.0105$ ) [16]. Venoocclusive disease (VOD) was observed in $11.6 \%$ of patients receiving inotuzumab [16]. Hematopoietic stem cell transplant was received by $43 \%$ of patients receiving inotuzumab and $11 \%$ of patients receiving SOC [16].

Song and colleagues conducted a matching-adjusted indirect comparison (MAIC) of blinatumomab versus inotuzumab in R/R B-cell precursor ALL using individual patient data (IPD) from TOWER and aggregate data from INOVATE-ALL [17, 18]. To be consistent with the inclusion criteria of INO-VATE-ALL (i.e., $\leq 1$ one prior salvage chemotherapy regimen), patients in TOWER receiving two or more prior salvage treatments were excluded from the MAIC. The remaining patients in TOWER were then weighted to match patients in INO-VATE-ALL on the following characteristics: age, sex, race, ECOG performance status, bone-marrow blast count, number of lines of previous salvage therapy, whether or not patients had previously received allo-SCT, rate of CR and $\mathrm{CR}$ with incomplete hematologic recovery (CRi) during the most recent induction therapy, and duration of first remission. After matching, the median OS was 9.3 months for blinatumomab and 7.7 months for inotuzumab (weighted log-rank test $p=0.4$ ). The $\mathrm{CR}$ rates for blinatumomab were similar before and after matching [17]. Because INO-VATEALL included $\mathrm{Ph}+$ patients whereas TOWER did not, it was not possible to match the two trials on this characteristic.

Information on the cost effectiveness of novel therapeutics is increasingly used by payers in their decision making regarding reimbursement for and access to such treatments. 
A previous study using data from TOWER and other sources concluded that, given an incremental cost-effectiveness ratio (ICER) threshold of US $\$ 150,000$ per quality-adjusted lifeyear (QALY) gained, blinatumomab is cost effective versus SOC chemotherapy in adult patients with $\mathrm{Ph}-\mathrm{R} / \mathrm{R}$ B-cell precursor ALL from a US healthcare payer perspective [19]. However, the cost effectiveness of blinatumomab versus inotuzumab has not been examined.

\section{Methods}

\subsection{Model Overview}

The objective of this study was twofold: (1) evaluate the cost effectiveness of blinatumomab versus inotuzumab in patients with R/R B-cell precursor ALL who have received one or no prior salvage therapy from a US healthcare payer perspective using data from INO-VATE-ALL and the MAIC-weighted subgroup of TOWER as reported by Song et al. [17, 18], and (2) examine the impact of different approaches for applying the results of the MAIC on results of the cost-effectiveness analysis. The analysis used a partitioned survival model with states defined on response, relapse, and death (Fig. 1). The model has a weekly cycle length and a 50-year time horizon approximating a lifetime projection [20]. The model takes as inputs probabilities of CR, survival distributions for event-free survival (EFS) for patients with CR, survival distributions for OS, duration of blinatumomab and inotuzumab treatment, probabilities of allo-SCT, general population mortality rates, utility values, and costs. Costs include those of drug acquisition and administration, adverse events (AEs), allo-SCT, subsequent salvage therapy, and end-of-life care, and were adjusted to 2018 prices using the consumer price index for medical care [21]. Patients entering the model were assumed to spend the first 12 weeks in the initial state (corresponding to the period over which response was assessed in TOWER), during which time they were only at risk of death, and were assigned initial (baseline) utility values. At the end of this 12-week initial period, patients who had achieved CR were assumed to enter the 'response' state, while the remaining patients entered the $R / R$ state where they remained until death. Patients in the 'response' state were assumed to be at risk of relapse or death. Additional details regarding the model structure can be found in the aforementioned cost-effectiveness analysis of blinatumomab versus SOC [19].

\subsection{Model Parameters}

Estimation of the model parameters is summarized in the following sections and in Tables 1 and 2 .

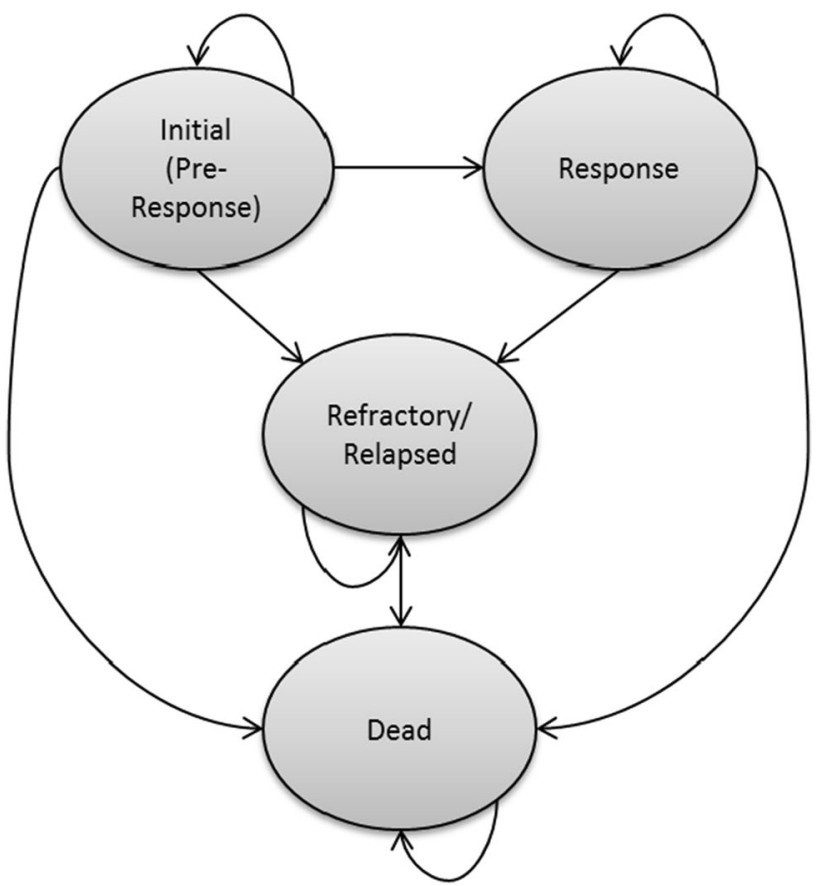

Fig. 1 Model schematic. Although transitions amongst states are shown in figure, the model employs partitioned survival approach. Transitions are therefore not modeled explicitly

\subsubsection{Alternative Approaches to Estimating the Effectiveness of Blinatumomab and Inotuzumab Based on Matching Adjusted Indirect Comparison (MAIC)}

A variety of different approaches may be considered when conducting analyses of cost effectiveness based on an indirect comparison of data from two or more controlled trials. These approaches include the use of anchored or unanchored comparisons. For anchored comparisons, different approaches may be taken with regard to the choice of the reference treatment to which measures of treatment effects are applied (no reference treatment need be specified for unanchored comparisons). Because the choice of the approach has the potential to impact the results of the analysis, several different approaches were used to estimate the effectiveness of blinatumomab and inotuzumab, in terms of probabilities of CR and OS, using the MAIC-adjusted data from TOWER, and published information from INO-VATEALL. Specifically, both anchored and unanchored comparisons were conducted. For the anchored comparisons, four different reference treatments were used, corresponding to the four treatment arms included in the TOWER and INOVATE-ALL trials (blinatumomab and SOC arms of TOWER and inotuzumab and SOC arms of INO-VATE-ALL). Thus, a total of five different approaches (four anchored and one unanchored) were used to estimate CR and OS, which are 


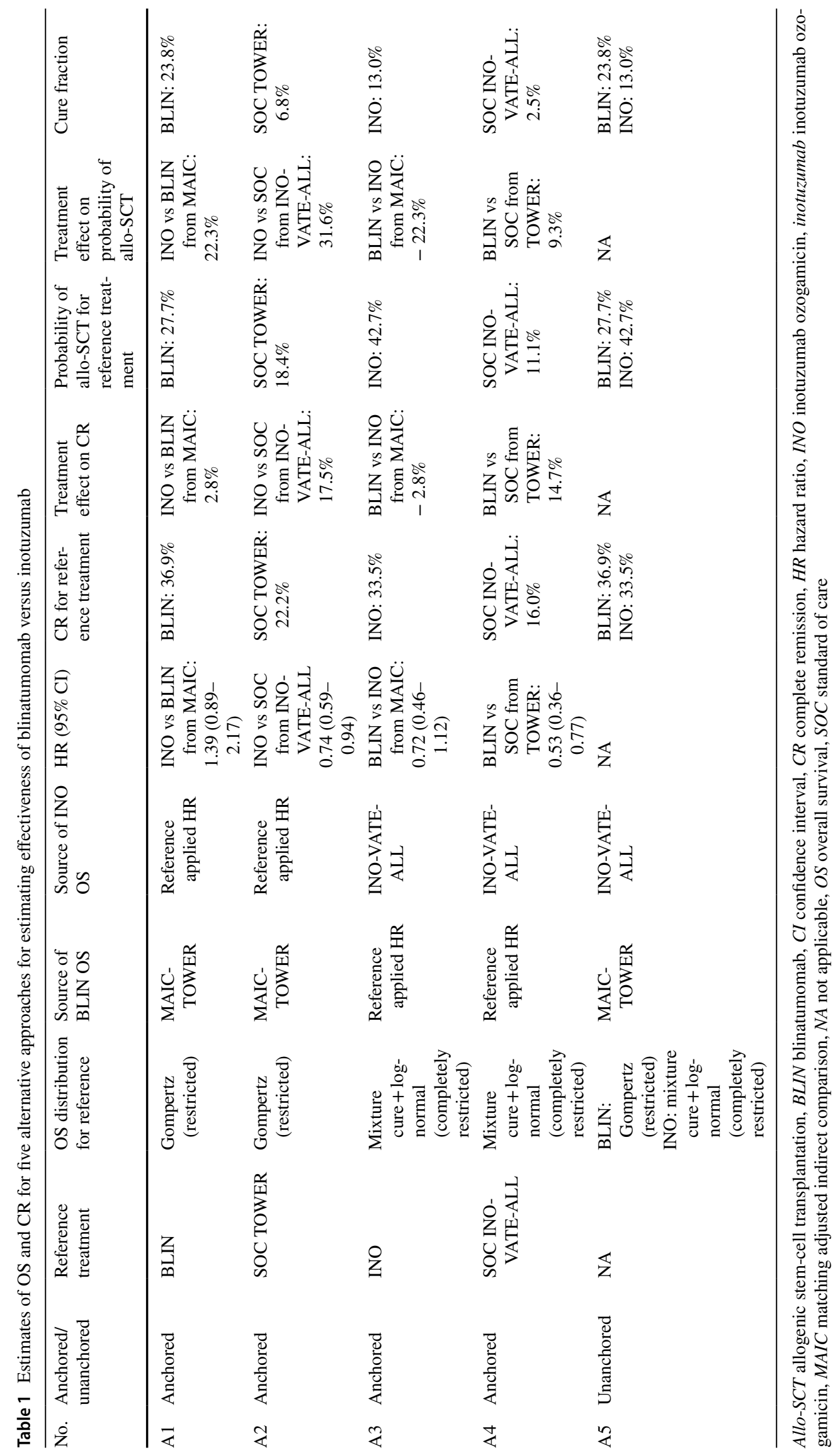


Table 2 Other model parameters

\begin{tabular}{|c|c|c|c|c|}
\hline Parameter & Point estimate & SE & Distribution & Source \\
\hline \multicolumn{5}{|l|}{ Analytic variables } \\
\hline Timeframe (years) & 50 & NA & Constant & Assumption \\
\hline Annual discount rate, costs (\%) & 3 & NA & Constant & Sanders et al. [39] \\
\hline Annual discount rate, effectiveness (\%) & 3 & NA & Constant & Sanders et al. [39] \\
\hline ICER threshold (US\$/QALY gained) & 150,000 & NA & Constant & Assumption \\
\hline \multicolumn{5}{|l|}{ Patient characteristics } \\
\hline Starting age (years) & 45.9 & NA & Bootstrap & TOWER after MAIC adjustment \\
\hline Percent male & 55.5 & NA & Bootstrap & TOWER after MAIC adjustment \\
\hline \multicolumn{5}{|l|}{ Costs } \\
\hline \multicolumn{5}{|l|}{ Medication costs } \\
\hline \multicolumn{5}{|l|}{ Blinatumomab } \\
\hline $\begin{array}{l}\text { Blinatumomab } 35 \mu \mathrm{g}(28 \mu \mathrm{g} \text { useable) } \\
\text { (US\$) }\end{array}$ & 3707 & NA & Constant & IBM Micromedex $^{\circledR}$ RED BOOK $^{\circledR}[35]$ \\
\hline \multicolumn{5}{|l|}{ Inotuzumab } \\
\hline Inotuzumab 0.9-mg vial (US\$) & 18,700 & NA & Constant & IBM Micromedex ${ }^{\circledR}$ RED BOOK ${ }^{\circledR}[35]$ \\
\hline $\begin{array}{l}\text { Average number of vials per course of } \\
\text { therapy }\end{array}$ & 9.49 & NA & Constant & NICE STA Committee Papers [34] \\
\hline \multicolumn{5}{|l|}{ Administration costs } \\
\hline \multicolumn{5}{|l|}{ Inpatient } \\
\hline \multicolumn{5}{|l|}{ Blinatumomab } \\
\hline \multicolumn{5}{|l|}{ Inpatient days per cycle received } \\
\hline Cycle 1 & 9 & NA & Constant & BLINCYTO prescribing information $[3,9]$ \\
\hline Cycle 2 & 2 & NA & Constant & BLINCYTO prescribing information $[3,9]$ \\
\hline Cycle 3+ & 0 & NA & Constant & BLINCYTO prescribing information $[3,9]$ \\
\hline Cost per inpatient day (US\$) & 6036 & 3704 & Lognormal & Barlev et al. $[21,31]$ \\
\hline \multicolumn{5}{|l|}{ Outpatient } \\
\hline \multicolumn{5}{|l|}{ Blinatumomab } \\
\hline Days per bag change & 2 & NA & Constant & Assumption \\
\hline $\begin{array}{l}\text { Cost per day of home infusion therapy } \\
\text { (US\$) }\end{array}$ & 68 & 17 & Lognormal & $\begin{array}{l}\text { Blue Cross and Blue Shield of Michigan. } \\
\text { Medicare Advantage PPO Enhanced Ben- } \\
\text { efits Fee Schedule [32] }\end{array}$ \\
\hline $\begin{array}{l}\text { Cost per outpatient visit, refill of infusion } \\
\text { pump (US\$) }\end{array}$ & 150 & 38 & Lognormal & $\begin{array}{l}\text { Center for Medicare and Medicaid Services } \\
\text { [33] }\end{array}$ \\
\hline \multicolumn{5}{|l|}{ Inotuzumab } \\
\hline Outpatient visits per cycle & 3 & NA & Constant & Kantarjian et al. [11] \\
\hline Median number of cycles & 3 & NA & Constant & Kantarjian et al. [11] \\
\hline $\begin{array}{l}\text { Cost per outpatient visit, IV infusion up } \\
\text { to } 1 \mathrm{~h} \text { (US\$) }\end{array}$ & 145 & 36 & Lognormal & $\begin{array}{l}\text { Center for Medicare and Medicaid Services } \\
\text { [33] }\end{array}$ \\
\hline \multicolumn{5}{|l|}{ Probability of VOD (\%) } \\
\hline Blinatumomab patients & 0.00 & 0.00 & Constant & TOWER after MAIC adjustment \\
\hline Inotuzumab patients & 11.59 & 2.50 & Beta & Kantarjian et al. [16] \\
\hline Cost of treatment of VOD (US\$) & 57,078 & 17,487 & Lognormal & Zhang et al. [21, 37] \\
\hline Cost of allo-SCT (US\$) & 396,861 & 38,277 & Lognormal & Zhang et al. $[21,38]$ \\
\hline \multicolumn{5}{|l|}{$\begin{array}{l}\text { Probability of receiving subsequent salvage } \\
\text { therapy }(\%)\end{array}$} \\
\hline \multicolumn{5}{|l|}{ Blinatumomab patients } \\
\hline Blinatumomab & 0.00 & NA & Constant & TOWER after MAIC adjustment \\
\hline Inotuzumab & 4.84 & NA & Bootstrap & TOWER after MAIC adjustment \\
\hline Multi-agent chemotherapy & 29.25 & NA & Bootstrap & TOWER after MAIC adjustment \\
\hline \multicolumn{5}{|l|}{ Inotuzumab patients } \\
\hline Blinatumomab & 4.84 & NA & Bootstrap & TOWER after MAIC adjustment \\
\hline
\end{tabular}


Table 2 (continued)

\begin{tabular}{lllll}
\hline Parameter & Point estimate & SE & Distribution & Source \\
\hline Inotuzumab & 0.00 & NA & Constant & TOWER after MAIC adjustment \\
$\quad$ Multi-agent chemotherapy & 29.25 & NA & Bootstrap & TOWER after MAIC adjustment \\
$\begin{array}{l}\text { Cost per course of subsequent salvage } \\
\text { therapy (US\$) }\end{array}$ & & & & \\
$\quad$ Blinatumomab & 288,679 & 72,170 & Lognormal & Model output \\
$\quad$ Inotuzumab & 178,765 & 44,691 & Lognormal & Model output \\
$\quad$ Multi-agent chemotherapy & 64,866 & 16,217 & Lognormal & Delea et al. [19, 21] \\
Cost of terminal care (US\$) & 26,528 & 563 & Lognormal & Chastek et al. [21, 42] \\
Utility values & & & & \\
Initial & 0.789 & 0.012 & Lognormal (Disutility) & TOWER after MAIC adjustment \\
Response & 0.815 & 0.014 & Lognormal (Disutility) & TOWER after MAIC adjustment \\
Relapse/refractory & 0.751 & 0.013 & Lognormal (Disutility) & TOWER after MAIC adjustment \\
Terminal decrement & 0.045 & 0.015 & Lognormal (Disutility) & TOWER after MAIC adjustment \\
\hline
\end{tabular}

allo-SCT allogeneic stem cell transplant, BSA body surface area, ICER incremental cost-effectiveness ratio, inotuzumab inotuzumab ozogamicin, $I V$ intravenous, MAIC matching adjusted indirect comparison, $N A$ not applicable, $Q A L Y$ quality-adjusted life-years, $S E$ standard error, VOD veno-occlusive disease

referred to hereafter as approaches A1 to A5 (Table 1). Table 2 in the Electronic Supplementary Material provides more information as to how OS was estimated for the five approaches. For anchored comparisons (A1-A4), treatment effects on OS were expressed as HRs, which were applied to the reference parametric survival distributions fit to the MAIC-adjusted IPD from TOWER or 'pseudo' IPD derived from reported Kaplan-Meier curves from INOVATE-ALL using the algorithm reported by Guyot et al. $[16,22]$. Treatment effects on the probabilities of CR and receipt of allo-SCT were expressed as the absolute betweengroup difference in the probabilities of CR and allo-SCT that were applied to reported probabilities of CR and allo-SCT from TOWER and INO-VATE-ALL. All estimates from TOWER, including the probabilities of and treatment effects on CR and allo-SCT, as well as the survival distributions and HRs for OS, were based on the MAIC-adjusted sample of TOWER from the analysis conducted by Song and colleagues [17].

For A1 and A2, the distribution of OS for blinatumomab was based on a parametric survival distribution fit to MAICadjusted IPD from TOWER, while the distribution of OS for inotuzumab was obtained by applying the anchor-based MAIC-adjusted HR for inotuzumab versus blinatumomab to the distribution of OS for blinatumomab (A1) or the HR for inotuzumab versus SOC from INO-VATE-ALL to the distribution of OS for SOC TOWER (A2). For A3 and A4, the distribution of OS for inotuzumab was based on a parametric survival distribution fit to 'pseudo' IPD from INO-VATEALL, while the distribution of OS for blinatumomab was obtained by applying the anchor-based MAIC-adjusted HR for blinatumomab versus inotuzumab to the distribution of OS for inotuzumab (A3) or applying the MAIC-adjusted HR for blinatumomab versus SOC from TOWER to the distribution of OS for SOC from INO-VATE-ALL (A4). For the unanchored comparison (A5), parametric survival distributions fit to the MAIC-adjusted OS for blinatumomab from TOWER and the reported OS for inotuzumab from INOVATE-ALL were used directly (i.e., without the application of treatment effect estimates).

The probabilities of CR and allo-SCT for blinatumomab and inotuzumab were estimated similarly. Because EFS was not measured in INO-VATE-ALL, it was not possible to conduct a meaningful comparison of the two treatments on this measure. Accordingly, EFS (conditioned on CR for patients receiving inotuzumab) was assumed to be equal to that for patients receiving blinatumomab.

It should be noted that A1 and A3, which use HRs for OS from the anchored MAIC, require that the assumption of proportionality of hazards for OS are met in both trials. In contrast, A2 requires only that this assumption is met in the INO-VATE-ALL trial while A4 requires only that this assumption is met in the TOWER trial.

Analyses of Schoenfeld residuals found no statistically significant evidence of non-proportionality of hazards for OS in either the MAIC-weighted subgroup of TOWER patients who had received one or no prior salvage therapy or in INO-VATE-ALL ( $p=0.939$ and $p=0.734$, respectively) [23].

By evaluating cost effectiveness using the five approaches outlined above, it is possible to assess how relaxing the assumption of proportionality of hazards in one or both trials impacts the results. While the assumption of proportionality is not required for either trial for the unanchored comparison (A5), this approach is subject to bias due to confounding by variables not matched in the MAIC, whereas the anchored 
comparisons are not susceptible to confounding, but are susceptible to effect modification by variables not matched by the MAIC [24].

\subsubsection{Overall Survival and Event-Free Survival}

Long-term OS was estimated by fitting parametric survival distributions to MAIC-adjusted IPD from TOWER and pseudo IPD derived from digitized Kaplan-Meier curves from INO-VATE-ALL $[25,26]$. A number of parametric distributions were considered, including exponential, Weibull, log-logistic, lognormal, Gompertz, gamma, and restricted cubic spline distributions. Mixture and non-mixture cure models were also considered [27]. For all cure models, the cure fraction was assumed to vary by treatment group. Several different approaches with respect to the parameterization of treatment effects in the survival distributions were considered. These included (1) 'unrestricted' models, in which all distributional parameters were allowed to differ by treatment; (2) 'restricted' models, in which only a single parameter (e.g., the shape parameter for a Weibull distribution) was allowed to differ by treatment; and for cure models (3) 'completely restricted' models, in which the only difference between the treatments was the cure fraction (i.e., all parameters of the latent distribution were assumed to be the same for the two treatments). Parametric survival distributions were selected based on the Bayesian information criterion (BIC) statistic, visual inspection of survival distributions, hazard functions, time-dependent HRs, diagnostic plots for the nature of treatment effects, and external face validity of long-term extrapolations versus long-term historical comparator data [3]. The Gompertz (restricted) distribution was used to estimate OS for blinatumomab and SOC from TOWER, based on its good statistical and visual fit to the Kaplan-Meier curve, as well as its good fit to a historical comparator study of adult patients with $\mathrm{Ph}-\mathrm{R} / \mathrm{R}$ B-precursor ALL. The mixture cure + lognormal (completely restricted) distribution was used to estimate OS for inotuzumab and SOC from INO-VATE-ALL based on its good statistical and visual fit. For approach A5, OS for blinatumomab was based on the Gompertz distribution for blinatumomab used in approaches A1 and A2, while the OS for inotuzumab was based on the mixture cure + lognormal (completely restricted) distribution used for inotuzumab in approaches A3 and A4. While this results in different parametric forms being used to model blinatumomab and inotuzumab, this was done intentionally as the purpose of the A5 approach was to explicitly consider the possibility that the shapes of the OS distributions were different for blinatumomab and inotuzumab. EFS among responders was estimated based on the generalized gamma distribution fit to MAIC-adjusted IPD from TOWER. Age- and sexmatched US general population mortality rates were applied additively to the estimated parametric survival distributions for EFS and OS in order to account for increasing non-ALL mortality with age. Kaplan-Meier and parametric survival distributions for OS and EFS used in the model are shown in Fig. 2. Additional details regarding the selection of parametric survival distributions are provided in the Electronic Supplementary Material.

\subsubsection{Utility Values}

Health-state-specific utility values were estimated using MAIC-weighted EORTC-8D utility values from TOWER, and generalized estimating equations regression with covariates for treatment group (blinatumomab versus SOC), health state (baseline, pre-response assessment, in response [CR], $\mathrm{R} / \mathrm{R}$ ), interaction of treatment group and health state, and time from death ( $\geq 1$ month, $<1$ month) $[19,28,29]$. Lacking published utility values from INO-VATE-ALL, healthstate-specific utility values for inotuzumab were assumed to be the same as for blinatumomab. Utility values for patients surviving $>4$ years were based on age- and sex-matched US general-population utility values [30].

\subsubsection{Cost of Blinatumomab and Inotuzumab Medication and Administration}

Costs of blinatumomab treatment were estimated using the same general approach as employed in the analysis of cost effectiveness of blinatumomab versus SOC based on TOWER [19]. The medication cost of blinatumomab was calculated by daily cost of a $38.5-\mu \mathrm{g}$ vial of blinatumomab by the average number of days of therapy. The latter was calculated using MAIC-adjusted estimates of the percentages of patients who initiated and completed each cycle of blinatumomab therapy in TOWER, assuming that patients discontinuing within a cycle would do so at the midpoint of the cycle. Daily costs of hospitalization for blinatumomab administration were based on the average hospitalization cost for chemotherapy administration among patients with relapsed ALL reported in a recent retrospective health insurance claims study [31]. Patients receiving blinatumomab were assumed to be hospitalized for the first 9 days of the first cycle and the first 2 days of the second cycle of treatment according to the US FDA-approved prescribing information [9]. Costs of home infusion therapy of blinatumomab and visits for pump refill were based on the Medicare fees for home infusion therapy and refill and maintenance of a portable infusion pump, respectively $[32,33]$.

Inotuzumab patients were assumed to receive an average of 9.49 vials per course of therapy [34]. It was assumed that inotuzumab would be administered on an outpatient basis, and that patients would receive inotuzumab for three cycles [11]. Administration costs for inotuzumab were based on the 
Fig. 2 Parametric survival distributions fit to OS and to EFS given response in TOWER and INO-VATE-ALL

\section{A MAIC-adjusted OS based on TOWER}

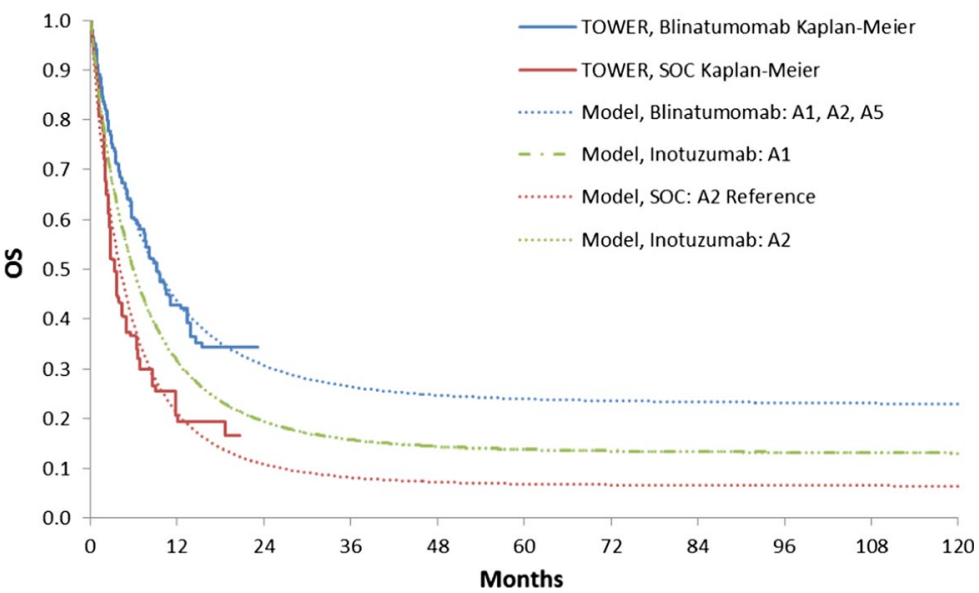

OS=Overall survival. EFS=Event-free survival. MAIC=Matching adjusted indirect comparison

\section{B OS based on INO-VATE-ALL}

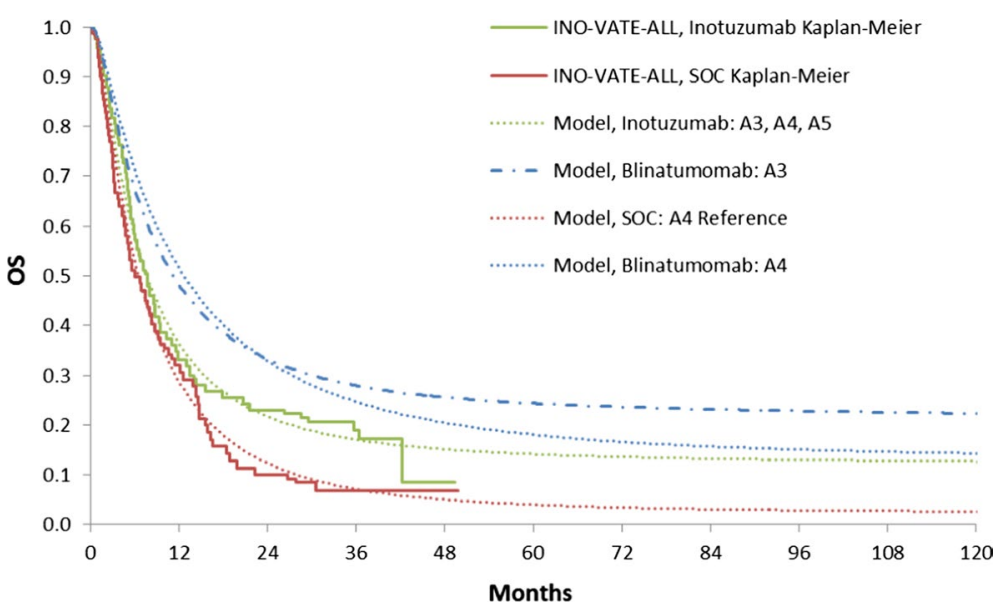

OS=Overall survival. SOC $=$ Standard of care

\section{MAIC-adjusted EFS given CR based on TOWER}

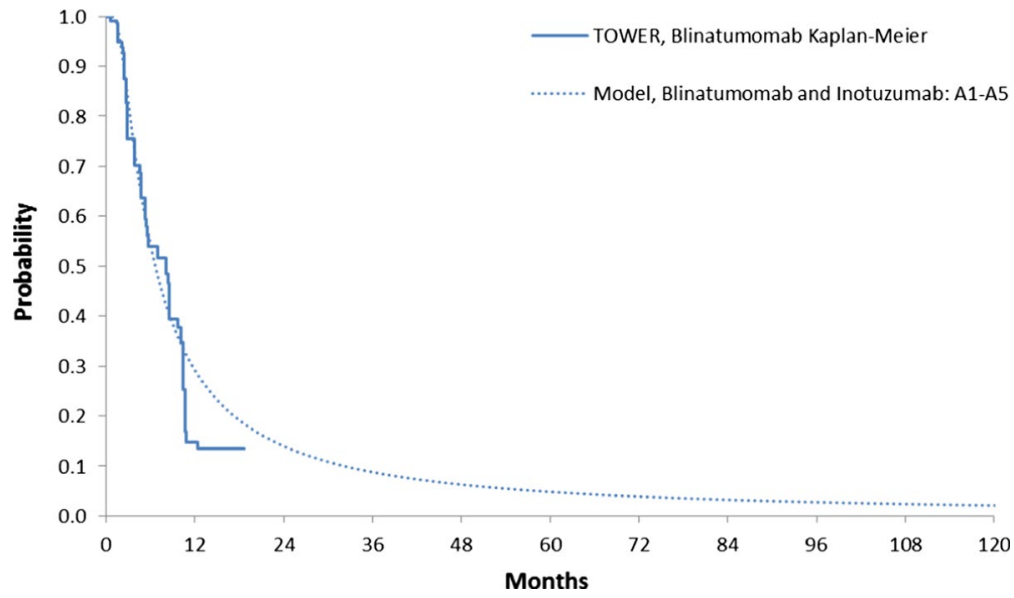

MAIC=Matching adjusted indirect comparison. EFS=Event-free survival. $C R=$ Complete remission. 
Medicare fee for an intravenous infusion of chemotherapy lasting up to $1 \mathrm{~h} \mathrm{[33].} \mathrm{Unit} \mathrm{costs} \mathrm{of} \mathrm{blinatumomab} \mathrm{and} \mathrm{ino-}$ tuzumab were based on wholesale acquisition cost (WAC) prices [35].

\subsubsection{Costs of Adverse Events}

The model includes the costs of treatment of CRS and VOD as these AEs are cited in the black box warnings in the US prescribing information for blinatumomab [9] and inotuzumab [14], respectively. Other AEs are not likely to have a material impact on incremental costs. Because most cases of CRS with blinatumomab would be observed during the phases of treatment when patients would be hospitalized (the median time to onset of CRS was 2 days after the start of infusion) [9], it was assumed that treatment of CRS would be captured in hospitalization costs for therapy administration. The impact on the ICER of including the cost of CRS management for blinatumomab patients was examined in a scenario analysis. In this scenario, the probability of CRS in patients receiving blinatumomab was estimated to be $3.9 \%$ based on the incidence of grade $\geq 3$ CRS in the MAIC-weighted subgroup of TOWER. The cost of CRS was estimated to be US\$27,738, based on the cost per day of treatment in an intensive care unit (US\$3719) [21, 36], the average duration of grade $\geq 3 \mathrm{CRS}$ in the MAIC-weighted subgroup of TOWER (6.6 days), and the cost of one dose of tocilizumab [35].

Because it was assumed that inotuzumab would be administered on an outpatient basis, the incremental cost of treatment of VOD was considered for patients receiving inotuzumab. The probability of VOD for patients receiving inotuzumab was based on the incidence of VOD of any grade observed in INO-VATE-ALL [16]. The cost of treatment of VOD was estimated to be US\$57,078, based on an estimate from a retrospective health-insurance claims study [21,37].

\subsubsection{Cost of Allogeneic Stem Cell Transplant}

The probabilities of allo-SCT were estimated based on the MAIC-adjusted proportion of patients undergoing allo-SCT during follow-up in TOWER $(27.7 \%$ and $18.4 \%$ for blinatumomab and SOC, respectively; absolute difference 9.3\%) and the proportion patients undergoing allo-SCT in the intent-to-treat (ITT) population in INO-VATE-ALL (42.7\% and $11.1 \%$, respectively; absolute difference $31.6 \%$ ). The cost of allo-SCT was estimated to be US $\$ 396,861$ based on the total healthcare costs in the 100 days after transplant in a retrospective health-insurance claims study of the economic burden of allo-SCT in patients with relapsed B-cell ALL in the US [21, 38].

\subsubsection{Cost of Subsequent Salvage Therapy}

Patients who do not achieve CR or who achieve CR but subsequently relapse were assigned the expected cost of subsequent salvage treatment with blinatumomab, inotuzumab, or SOC chemotherapy upon determination of no response or relapse. Patients initially receiving blinatumomab were assumed to receive salvage therapy with either inotuzumab or chemotherapy, whereas those initially receiving inotuzumab were assumed to receive salvage therapy with blinatumomab or chemotherapy. Because data from INO-VATEALL on subsequent salvage therapy were unavailable, it was assumed that the probabilities of receiving subsequent salvage chemotherapy would be the same for patients initially receiving inotuzumab as for those initially receiving blinatumomab, which was based on data for the MAIC-weighted sample of TOWER. The cost of subsequent salvage chemotherapy was based on the estimate used in the published cost-effectiveness analysis of TOWER [19, 21]. The cost per course of blinatumomab and inotuzumab as subsequent salvage therapy was assumed to be the same as that for initial treatment.

\subsection{Model Validation}

Analyses were conducted to test the model calculations including setting the discount rates for costs and effectiveness to $0 \%$ and ensuring that undiscounted and discounted life-years (LYs), QALYs, and costs are equal; setting utility values to 1.0 and ensuring that LYs equal QALYs; setting the model starting age to 50 years old, all patients to male, and OS to $100 \%$; and confirming that LYs were equal to life expectancy of a 50-year-old male in the US. Additionally, unit costs (medication, hospitalization, other administration costs, allo-SCT, and subsequent salvage therapy) and utility values were back-calculated from the model results. Results of sensitivity analyses were systematically checked against expectations. The results generated by the probabilistic sensitivity analysis (PSA) were compared with the deterministic results to ensure that the mean costs, QALYs, and ICER generated by the PSA were similar to those generated by the model. Model results were checked against results of other models (e.g., model used to evaluate cost effectiveness of blinatumomab versus SOC) to ensure consistency.

\subsection{Analyses}

For each of the five approaches for estimating effectiveness, life expectancy (LYs), QALYs, and expected lifetime costs were reported by treatment and health state (initial, CR, and R/R). LYs, QALYs, and costs were discounted at $3 \%$ annually [39]. Net monetary benefit (NMB) was calculated for a threshold value for cost effectiveness of US $\$ 150,000$ 
per QALY gained. Scenario analyses and deterministic sensitivity analyses (DSAs) were conducted to assess the robustness of findings (expressed as NMB) with respect to changes in key parameter estimates and assumptions. DSAs were reported as a tornado diagram. PSAs were conducted based on 10,000 Monte Carlo simulations. To ensure the correlation of parameter estimates across simulations, the probabilities of CR and allo-SCT, age at initiation of therapy, percent male patients at initiation of therapy, percent of blinatumomab patients starting and completing each cycle in TOWER, the percent of blinatumomab patients receiving subsequent salvage therapy, and parameters of the survival distributions for EFS and OS were sampled from a joint bootstrap or 'empirical' distribution, consisting of 1000 sets of bootstrap statistics derived from 1000 bootstrap samples of the TOWER data set. Cost-effectiveness acceptability curves for blinatumomab were then calculated.

\section{Results}

\subsection{Base Case}

Base-case results for the five approaches for estimating the effectiveness of blinatumomab and inotuzumab are presented in Table 3. In all analyses, blinatumomab was more costly and yielded more LYs and QALYs than inotuzumab. Incremental costs ranged from US\$7023 (A3) to US $\$ 36,244$ (A5). Incremental LYs ranged from 0.69 (A4) to 2.22 (A2). Incremental QALYs ranged from 0.54 (A4) to 1.78 (A1, A2). The ICER was below the threshold of US $\$ 150,000$ per QALY gained for blinatumomab versus inotuzumab with all five approaches, ranging from US\$4006 (A3) to US\$20,737 per QALY gained (A5). At a willingness-to-pay threshold of US\$150,000 per QALY gained, the NMB of blinatumomab versus inotuzumab ranged from US\$73,035 (A4) to US\$260,369 (A2).

\subsection{Sensitivity Analyses}

Given the similarity of results for several of the approaches, results of the DSAs, scenario analyses, and PSAs were only reported for approach A4 (anchored comparison using the SOC arm of INO-VATE-ALL as the reference), which was the least favorable for blinatumomab among the anchored approaches, and A5 (unanchored comparison). Results of the remaining scenarios (A1-A3) are reported in the Electronic Supplementary Material.

For both approaches for estimating the relative effectiveness of blinatumomab and inotuzumab, the model was most sensitive to the cost per day of hospitalization, duration of treatment with blinatumomab, and the number of vials of inotuzumab used (Fig. 3).
Alternative parametric distributions for OS were tested in scenarios 1 and 2. For scenario 1, the distributions for OS with the best visual and statistical fit that yielded more favorable results for blinatumomab versus the base case were selected (non-mixture cure Weibull (completely restricted) distribution for TOWER and non-mixture cure lognormal (completely restricted) distribution for INO-VATE-ALL). Similarly, for scenario 2, distributions that were less favorable to blinatumomab than in the base case, but still had good visual and statistical fit, were used. For TOWER, the nonmixture cure lognormal (completely restricted) distribution was used. For INO-VATE-ALL, the distribution selected for the base case [mixture cure lognormal (completely restricted)] already produced the least favorable results for blinatumomab among the plausible parametric OS distributions, and therefore no unfavorable scenario was available. Using 'favorable' parametric OS distributions (Scenario 1), the ICERs were US\$4862 per QALY gained for A4 and US\$9278 per QALY gained for A5 (Table 4). Using an 'unfavorable' OS distribution for blinatumomab in A5 (Scenario 2), the ICER was US $\$ 359,680$ per QALY gained. Additionally, a scenario was run for the A5 approach, in which the OS distribution for blinatumomab and inotuzumab arms was estimated simultaneously (Scenario 3 ). The nonmixture cure lognormal (completely restricted) model was selected for this scenario because it had the best visual fit to the Kaplan-Meier curves and was in the top 10 best fitting distributions in terms of statistical fit. The ICER for this scenario was US\$13,714 per QALY gained. More information on the selection of parametric distributions is available in the Electronic Supplementary Material.

Assuming patients surviving $>4$ years after treatment initiation would experience a four-fold increase in long-term mortality relative to the age- and sex-matched general population due to exposure to chemotherapy and allo-SCT and disease-related complications (Scenario 4) [40], the ICER was US\$17,289 per QALY gained for A4 and US\$28,994 per QALY gained for A5. Assuming long-term utility values for all patients would be reduced by 0.03 due to these factors (Scenario 5) with this decrement estimated as onehalf of the difference between the age- and sex-matched general population utility value at therapy initiation and the utility for patients with CR in TOWER, the ICERs were US\$15,532 per QALY gained for A4 and US\$21,387 per QALY gained for A5. When these assumptions were combined, the ICERs were US\$17,528 per QALY gained and US\$29,845 per QALY gained, respectively (Scenario 6). The ICERs for A4 and A5 were US\$124,702 per QALY gained and US\$54,676 per QALY gained, respectively, when it was assumed that the probability of allo-SCT for patients receiving inotuzumab would be equal to those receiving blinatumomab (Scenario 7). In the scenario where patients receiving blinatumomab would have to change their bags 


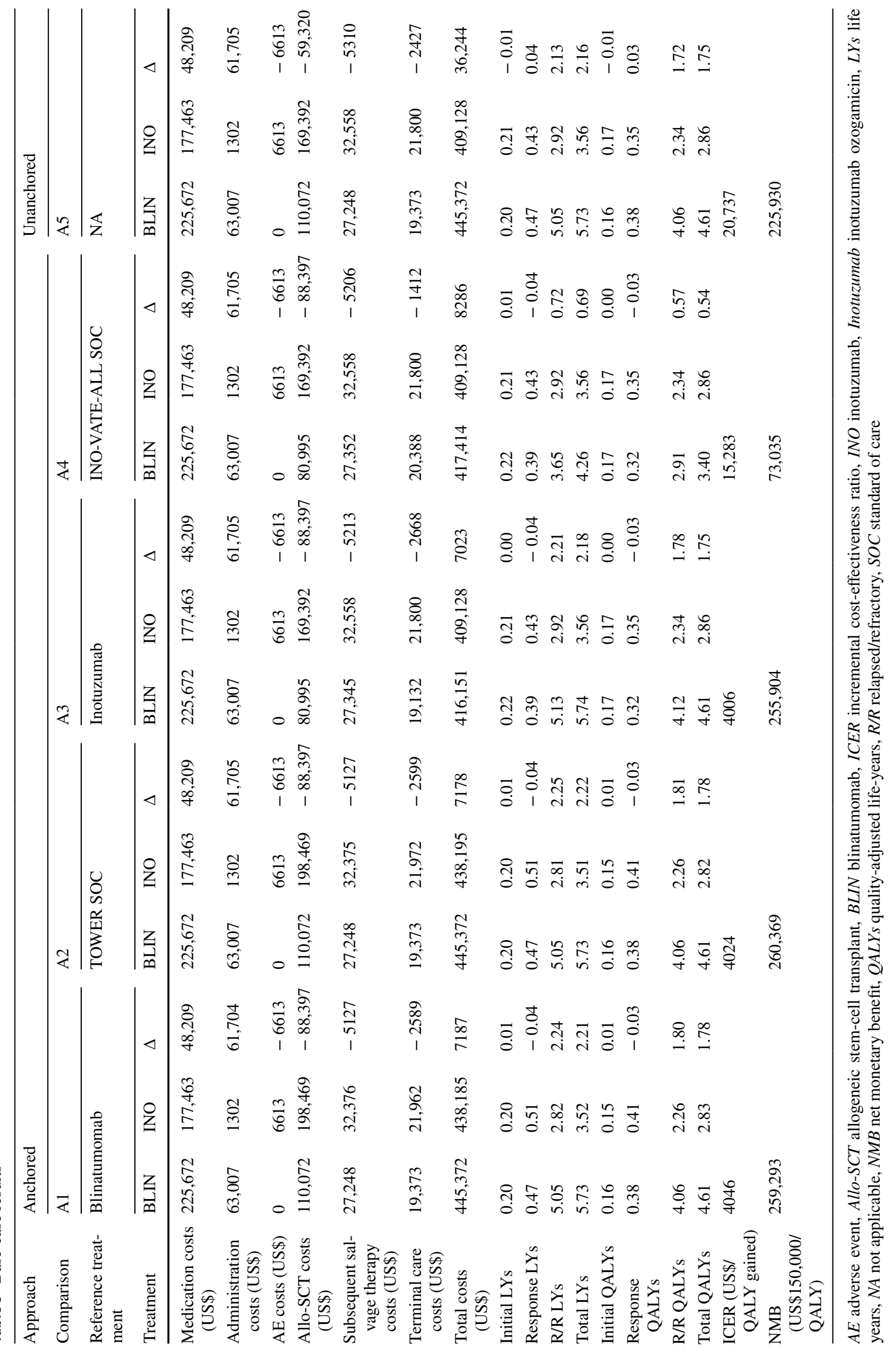


only once weekly (Scenario 8), the ICERs were US $\$ 4584$ per QALY gained and US\$17,418 per QALY gained for A4 and $\mathrm{A} 5$, respectively. Blinatumomab was dominant (less costly and more effective) compared with inotuzumab for both A4 and A5 when it was assumed that patients receiving inotuzumab would require 11.23 days in hospital to initiate treatment (Scenario 9) [34]. The ICERs for A4 and A5 were $\$ 17,265$ per QALY gained and $\$ 21,351$ per QALY gained, respectively, when incremental costs for CRS in patients receiving blinatumomab were included (Scenario 10).

The probability that blinatumomab is cost effective versus inotuzumab at an ICER threshold of US\$150,000 per QALY was $53.5 \%$ for A4 and $72.6 \%$ for A5 (Fig. 4).

\section{Discussion}

This study evaluated the cost effectiveness of blinatumomab versus inotuzumab in adult patients with $\mathrm{R} / \mathrm{R} \mathrm{B}$-cell precursor ALL receiving one or no prior salvage therapy from a US healthcare sector perspective based on data from INOVATE-ALL and the subgroup of patients in TOWER receiving one or no prior salvage who were weighted to match the reported characteristics of patients in INO-VATE-ALL using MAIC. Regardless of the approach employed to estimate the effectiveness of blinatumomab and inotuzumab, blinatumomab was cost effective versus inotuzumab, with ICERs ranging from US\$4006 to US\$20,737 per QALY gained. To the best of our knowledge, this is the first study to compare the cost effectiveness of blinatumomab versus inotuzumab in this setting.

This study is important as it highlights the complexities of incorporating indirect comparisons into economic evaluations and how a variety of different approaches may be employed, which may impact model results and conclusions. While in this study the ICERs for blinatumomab versus inotuzumab were favorable for all the approaches considered, similar results may not be obtained in all instances. Investigators conducting economic evaluations based on indirect comparisons should provide a rationale for the specific approach that they employ with respect to the choice of anchored versus unanchored comparisons and, for anchored comparisons, the reference treatment to which estimated treatment effects are applied. The impact of alternative approaches on model results should be examined in sensitivity analyses.

While all of the approaches employed for modeling survival for blinatumomab versus inotuzumab have their merits, approach A2 has some advantages over the others. Because approach A5 is based on an unanchored comparison, whereas approaches A1-A4 are based on anchored comparisons, the former is potentially biased by confounding and therefore is less preferred than the latter. Because approaches
A1 and A3 require the assumption of proportional hazards for both TOWER and INO-VATE-ALL, whereas approaches $\mathrm{A} 2$ and $\mathrm{A} 4$ require the proportional hazards assumption for only one of the two trials, A2 and A4 can be considered preferable to A1 and A3. The choice between A2 and A4 depends on the likely precision of the estimates. Although the number of subjects enrolled was greater in TOWER than INO-VATE-ALL, the duration of follow-up for OS was greater in INO-VATE-ALL than TOWER. An additional advantage of approach A2 is that the data on EFS and utilities were based on TOWER (as such data were unavailable from INO-VATE-ALL) and hence the data used in this approach are internally consistent. Based on this assessment, A2 can be considered the preferred approach amongst all those considered.

Interestingly, although the rate of $\mathrm{CR}$ was higher with blinatumomab than SOC in the ITT patient population in TOWER, the unadjusted rate of allo-SCT was similar among the two treatment arms. This is in contrast with INO-VATEALL in which there were differences in both the response rate and the rate of allo-SCT. The precise reason for this finding is uncertain, as the decision to undergo transplant is made by the patients and their physicians, and utilization of allo-SCT varies considerably by setting. However, approximately $10 \%$ of patients receiving blinatumomab received maintenance therapy with blinatumomab, which may have been used as an alternative to allo-SCT in TOWER (there was no maintenance therapy with inotuzumab in INOVATE-ALL). Also, patients in TOWER had more advanced ALL than those in INO-VATE-ALL, and were more likely to have undergone prior SCT, potentially also attenuating differences between groups in the transplant rate relative to what was observed among the less advanced patients in INO-VATE-ALL. Although the rate of allo-SCT was the same for both TOWER groups in the ITT population (24\%), the MAIC-adjusted rates were $27.7 \%$ and $18.4 \%$ for blinatumomab and SOC, respectively (compared with MAICadjusted rates of CR of $36.9 \%$ and $22.2 \%$, respectively).

Although the estimated rate of allo-SCT was higher with inotuzumab in INO-VATE-ALL than with blinatumomab in the MAIC-adjusted population of TOWER, results of the analyses presented here suggest that OS is greater with blinatumomab than with inotuzumab. This counter-intuitive finding, given the prognostic significance of allo-SCT in this population, may be explained by two factors. First, the TOWER study showed a clear and statistically significant benefit on OS in the absence of any difference between arms in the observed rate of allo-SCT, suggesting the benefits of blinatumomab are independent of receipt of transplant. Second, data from INO-VATE-ALL indicate an increased risk of post-transplant non-relapse mortality in patients receiving inotuzumab, suggesting the benefit of transplant may be modified by receipt of inotuzumab treatment. 
Fig. 3 Tornado diagrams on NMB for blinatumomab versus inotuzumab

\section{A Anchored/SOC INO-VATE-ALL=Reference (A4)}

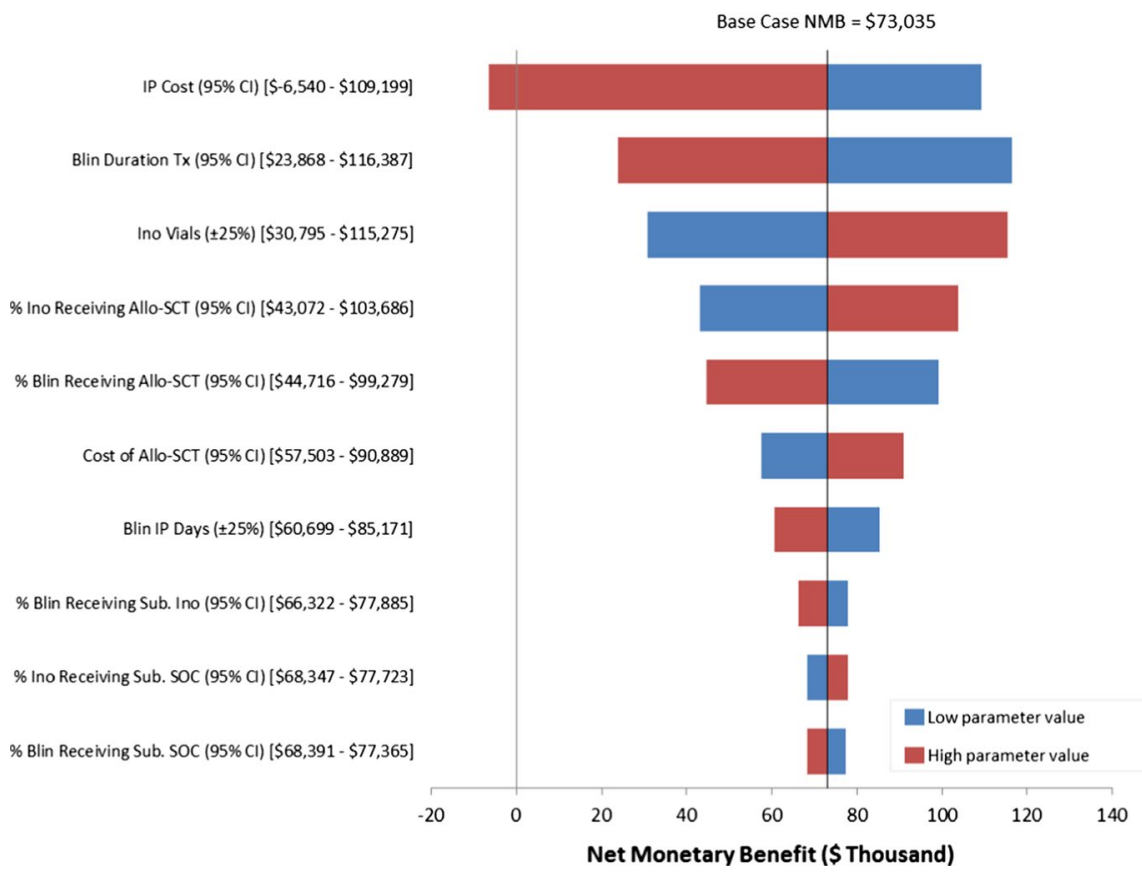

$\mathrm{NMB}=$ Net monetary benefit. $\mathrm{SOC}=$ Standard of care. Blin=Blinatumomab. Ino=Inotuzumab. Allo$\mathrm{SCT}=$ Allogeneic stem cell transplant. IP=Inpatient. R/R=Relapsed/refractory. Sub.=Subsequent

\section{B Unanchored (A5)}

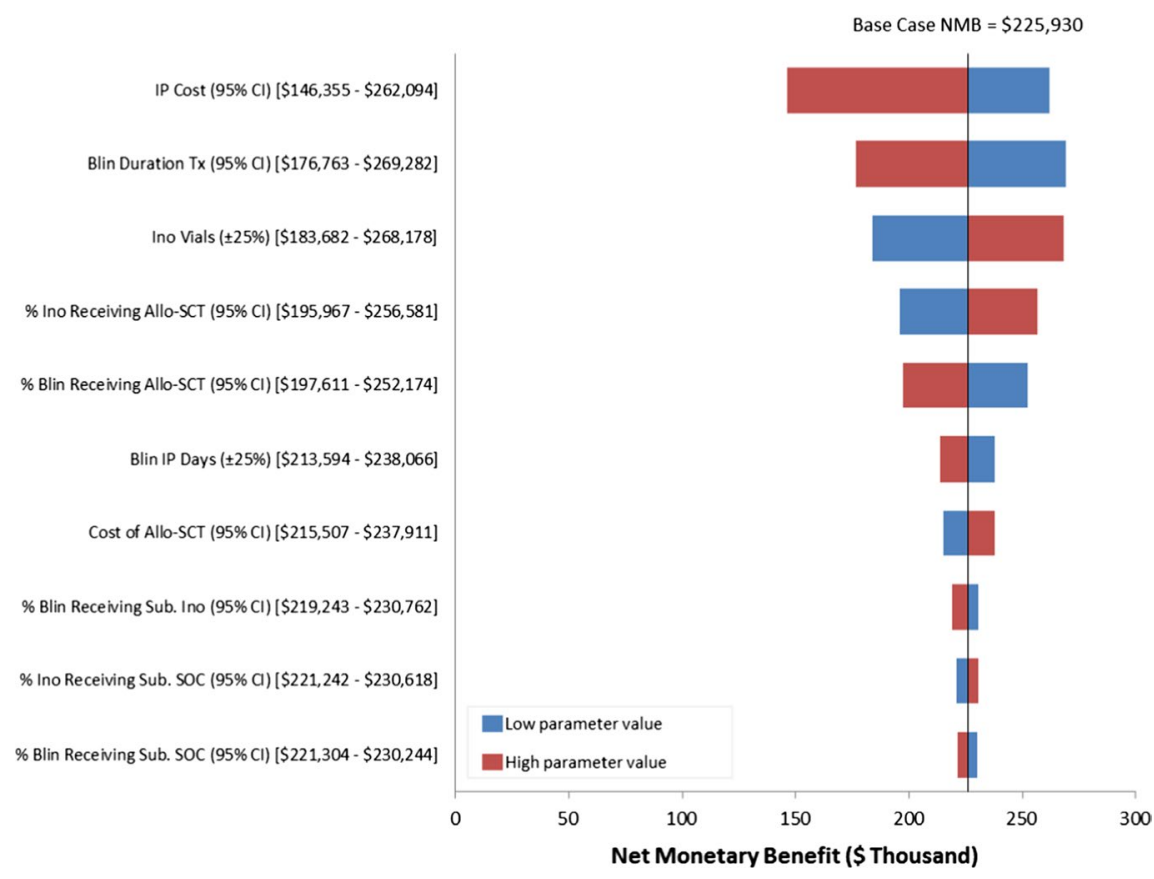

Blin=Blinatumomab. Tx=Treatment. Ino=Inotuzumab. Allo-SCT=Allogeneic stem cell transplant. $\mathrm{IP}=$ Inpatient. R/R=Relapsed/refractory. Sub.=Subsequent. SOC=Standard of care . 
Table 4 Scenario analyses

\begin{tabular}{|c|c|c|c|c|c|c|c|}
\hline \multirow[t]{2}{*}{ No. } & \multirow[t]{2}{*}{ Scenario } & \multicolumn{3}{|c|}{$\begin{array}{l}\text { A4: anchored comparison/INO-VATE-ALL } \\
\text { SOC }=\text { reference }\end{array}$} & \multicolumn{3}{|c|}{ A5: unanchored comparison } \\
\hline & & $\begin{array}{l}\text { Incremental } \\
\text { cost (US\$) }\end{array}$ & $\begin{array}{l}\text { Incremental } \\
\text { QALYs }\end{array}$ & $\begin{array}{l}\text { ICER (US\$/ } \\
\text { QALY gained) }\end{array}$ & $\begin{array}{l}\text { Incremental } \\
\text { cost (US\$) }\end{array}$ & $\begin{array}{l}\text { Incremental } \\
\text { QALYs }\end{array}$ & $\begin{array}{l}\text { ICER (US\$/ } \\
\text { QALY } \\
\text { gained) }\end{array}$ \\
\hline & Base case & 8286 & 0.54 & 15,283 & 36,244 & 1.75 & 20,737 \\
\hline 1 & Favorable OS distribution & 7180 & 1.48 & 4862 & 33,776 & 3.64 & 9278 \\
\hline 2 & Unfavorable OS distribution $^{\mathrm{a}}$ & NA & NA & NA & 37,228 & 0.10 & 359,680 \\
\hline 3 & Same OS distributions & NA & NA & NA & 35,070 & 2.56 & 13,714 \\
\hline 4 & $4 \times$ increase in general population mortality & 8241 & 0.48 & 17,289 & 36,268 & 1.25 & 28,994 \\
\hline 5 & 0.03 disutility for long-term survivors & 8286 & 0.53 & 15,532 & 36,244 & 1.69 & 21,387 \\
\hline 6 & $\begin{array}{l}4 \times \text { increase in general population mortality } \\
\text { and } 0.03 \text { disutility for long-term survivors }\end{array}$ & 8241 & 0.47 & 17,528 & 36,268 & 1.22 & 29,845 \\
\hline 7 & Allo-SCT rate equal for BLIN and INO & 67,606 & 0.54 & 124,702 & 95,564 & 1.75 & 54,676 \\
\hline 8 & 7 days per bag outpatient BLIN & 2485 & 0.54 & 4584 & 30,444 & 1.75 & 17,418 \\
\hline 9 & $\begin{array}{l}\text { Inpatient days for INO based on NICE ERG } \\
\text { recommendation ( } 11.23 \text { days) }\end{array}$ & $-55,011$ & 0.54 & Dominant & $-27,065$ & 1.75 & Dominant \\
\hline 10 & Include cost of CRS management & 9360 & 0.54 & 17,265 & 37,318 & 1.75 & 21,351 \\
\hline
\end{tabular}

Allo-SCT allogeneic stem cell transplant, BLIN blinatumomab, CRS cytokine release syndrome, ERG evidence review group, ICER incremental cost effectiveness ratio, INO inotuzumab ozogamicin, $N A$ not applicable, NICE National Institute of Health and Care Excellence, $O S$ overall survival, $Q A L Y s$ quality-adjusted life-years, $S O C$ standard of care, $Q A L Y$ quality-adjusted life-year, $S O C$ standard of care

${ }^{a}$ The mixture cure + lognormal (completely restricted) distribution used for modeling OS in INO-VATE-ALL in the base case was the most favorable distribution for inotuzumab. Accordingly, no 'unfavorable' distribution was analyzed for A4

Fig. 4 Cost-effectiveness acceptability curves for blinatumomab versus inotuzumab

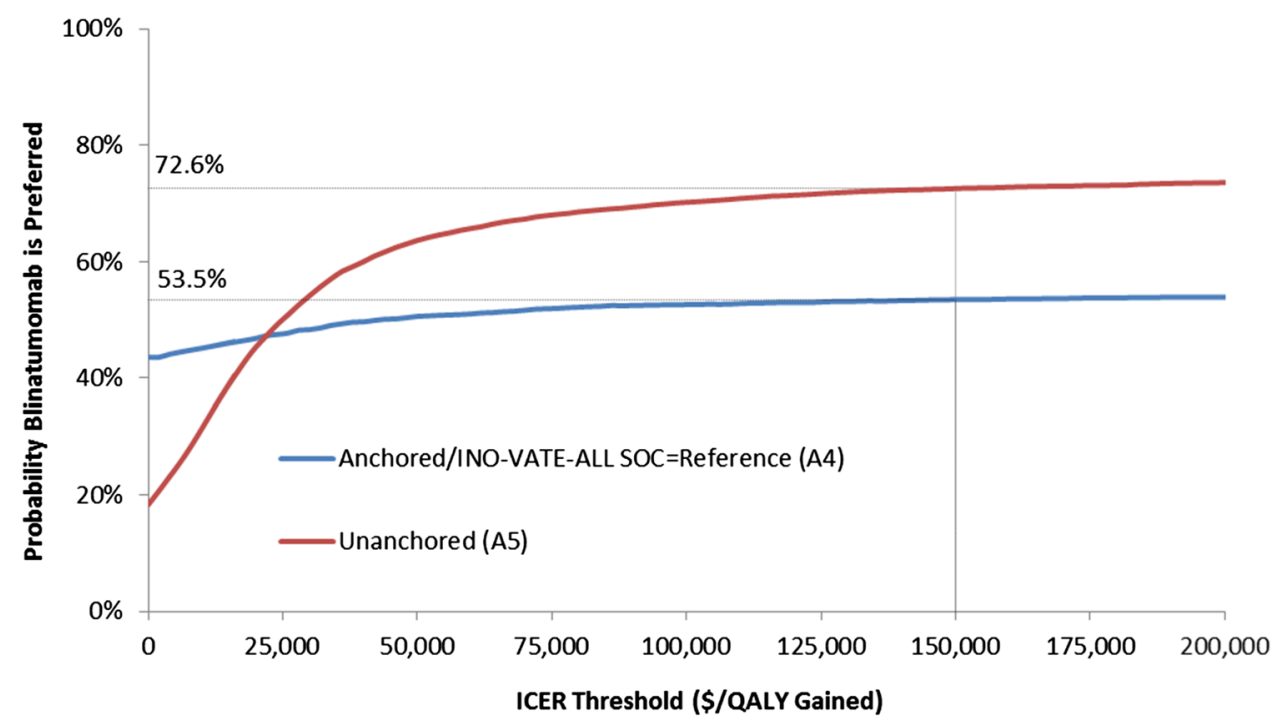

SOC $=$ Standard of care. ICER=Incremental cost-effectiveness ratio.
Limitations of this evaluation should be considered when interpreting these results. First, although patients in TOWER were matched to those in INO-VATE-ALL using MAIC, there may be other characteristics that were not reported for INO-VATE-ALL that may have differed between trials and which may have biased the comparison.
Second, because INO-VATE-ALL included both $\mathrm{Ph}+$ and $\mathrm{Ph}-$ patients (15\% and $85 \%$, respectively), whereas TOWER included only $\mathrm{Ph}$ - patients, it was necessary to assume that $\mathrm{Ph}$ status is not prognostic for $\mathrm{CR}, \mathrm{OS}$, or receipt of allo-SCT (for the unanchored comparison) and does not modify the effect of blinatumomab treatment on these 
outcomes (for both anchored and unanchored comparisons) when generalizing the results from the TOWER trial to the population of INO-VATE-ALL. Although prognosis is generally considered to be worse for patients with $\mathrm{Ph}+$ versus $\mathrm{Ph}-\mathrm{ALL}$ [41], that was not the case in INO-VATE-ALL (median OS 8.7 and 8.4 months for inotuzumab and SOC, respectively, in the $\mathrm{Ph}+$ population and 7.7 and 6.2 months, respectively, in the overall population) [16]. Because data on outcomes from controlled trials of blinatumomab versus SOC in patients with $\mathrm{Ph}+\mathrm{ALL}$ and characteristics similar to those in INO-VATE-ALL are unavailable, the effect of $\mathrm{Ph}$ status on the efficacy of blinatumomab in such patients is unknown. The impact of this limitation on results of this analysis is therefore uncertain.

Third, the effectiveness and costs of treatment with blinatumomab and inotuzumab in typical US clinical practice may differ from that observed in the TOWER and INOVATE-ALL trials. In particular, the rates of hospitalization for administration of treatment, frequency of allo-SCT, and utilization of blinatumomab as maintenance therapy are likely to vary across settings. Differences in these factors may materially affect the relative costs and effectiveness of these treatments.

Stelljes et al. reported results of an MAIC of inotuzumab versus blinatumomab and concluded that inotuzumab has an advantage over blinatumomab with respect to CR/CRi, EFS, and OS [41]. Because INO-VATE-ALL did not enroll patients with two or more prior salvage therapies, and because patient characteristics and endpoint results for the subgroup of patients in TOWER with one or no prior salvage therapy have not been reported, Stelljes and colleagues were unable to match the INO-VATE-ALL patients with those in TOWER on this characteristic. Line of salvage therapy is an important negative prognostic factor, and the treatment effect on OS for blinatumomab versus SOC was nominally greater in patients with one or no versus two or more prior salvage therapies [7]. The inability to match on this characteristic, due to lack of data, therefore may have biased both their unanchored and anchored comparisons in favor of inotuzumab. Also, while Stelljes et al. correctly note that the OS curves in the overall population of TOWER converged at the end of follow-up, this phenomenon was not observed in the MAIC-adjusted subgroup of TOWER patients with one or no prior salvage therapy. Their conclusions regarding the HRs for blinatumomab versus inotuzumab over time therefore may not be applicable in the population examined in our analysis.

\section{Conclusions}

For various approaches for estimating the relative effectiveness of blinatumomab versus inotuzumab, blinatumomab was estimated to be cost effective compared with inotuzumab in adult patients with R/R B-cell precursor ALL with one or no prior salvage therapy from a US payer perspective.

Data Availability Statement The authors declare that all input data to parameterize the decision analytic model are available within the article and the Electronic Supplementary Material. The model can be re-built entirely based on the information provided.

\section{Compliance with Ethical Standards}

Funding Support for this study was provided by Amgen, Inc., Thousand Oaks, CA, USA. The authors were responsible for all content and editorial decisions, and they received no honoraria related to the development of this publication.

Competing interests TED is an employee and owner of PAI. PAI received research funding from Amgen for this study. PAI has received research funding and consulting fees from Amgen and Pfizer for research activities unrelated to this study. $\mathrm{XZ}$ was an employee at the time this study was conducted and is a stockholder of Amgen. JA is an employee of PAI. DB is an employee of PAI. FD is an employee and stockholder of Amgen. MC is an employee and stockholder of Amgen. $\mathrm{ZC}$ was an employee at the time this study was conducted and is a stockholder of Amgen.

Authors' Contributions TED: Study design, data collection, data analysis, interpretation of results, writing of draft and final manuscript. XZ: Study design, data collection, data analysis, interpretation of results, writing of draft and final manuscript. JA: Study design, data collection, data analysis, interpretation of results, writing of draft and final manuscript. DB: Study design, data collection, data analysis, interpretation of results, writing of draft and final manuscript. FD: Study design, interpretation of results, writing of draft and final manuscript. MC: Study design, interpretation of results, writing of draft and final manuscript. ZC: Study design, data collection, data analysis, interpretation of results, writing of draft and final manuscript. All authors contributed to the drafting and revision of the manuscript, and approved it for submission. TED is the principal investigator and will act as total guarantor for the study.

Open Access This article is distributed under the terms of the Creative Commons Attribution-NonCommercial 4.0 International License (http://creativecommons.org/licenses/by-nc/4.0/), which permits any noncommercial use, distribution, and reproduction in any medium, provided you give appropriate credit to the original author(s) and the source, provide a link to the Creative Commons license, and indicate if changes were made. 


\section{References}

1. Terwilliger T, Abdul-Hay M. Acute lymphoblastic leukemia: a comprehensive review and 2017 update. Blood cancer journal. 2017;7(6): e577.

2. National Cancer Institute Surveillance Epidemiology and End Results Program. SEER Cancer Stat Facts: Acute Lymphocytic Leukemia. National Cancer Institute. Bethesda, MD. https://seer. cancer.gov/statfacts/html/alyl.html. Accessed 29 June 2018.

3. European Medicines Agency. Blincyto (blinatumomab) European Public Assessment Report. http://www.ema.europa.eu/docs/en GB/document library/EPAR - Public assessment report/human /003731/WC500198227.pdf. Accessed 22 June 2018. 2015.

4. Frey NV, Luger SM. How I treat adults with relapsed or refractory Philadelphia chromosome-negative acute lymphoblastic leukemia. Blood. 2015;126(5):589-96.

5. National Comprehensive Cancer Network. NCCN Clinical Practice Guidelines in Oncology. Acute Lymphoblastic Leukemia. Version 1.2018-March 12, 2018. https://www.nccn.org/profe ssionals/physician_gls/default.aspx\#all. Accessed 18 Apr 2018.

6. Gokbuget N, Dombret H, Ribera JM, Fielding AK, Advani A, Bassan $\mathrm{R}$, et al. International reference analysis of outcomes in adults with B-precursor Ph-negative relapsed/refractory acute lymphoblastic leukemia. Haematologica. 2016;101(12):1524-33.

7. Kantarjian H, Stein A, Gokbuget N, Fielding AK, Schuh AC, Ribera JM, et al. Blinatumomab versus Chemotherapy for Advanced Acute Lymphoblastic Leukemia. N Engl J Med. 2017;376(9):836-47.

8. Food and Drug Administration. FDA approves Blincyto to treat a rare form of acute lymphoblastic leukemia. http://www.fda.gov/ NewsEvents/Newsroom/PressAnnouncements/ucm425549.htm. Accessed 22 Dec 2015

9. Amgen. BLINCYTO ${ }^{\circledR}$ (blinatumomab) for injection, for intravenous use [full prescribing information]. Initial U.S. Approval: 2014. Revised: 5/2018. http://pi.amgen.com/united_states/blinc yto/blincyto_pi_hcp_english.pdf. Accessed 30 May 2018.

10. Topp MS, Zimmerman Z, Cannell P, Dombret H, Maertens J, Stein A, et al. Health-related quality of life in adults with relapsed/ refractory acute lymphoblastic leukemia treated with blinatumomab. Blood. 2018;131(26):2906-14.

11. Kantarjian HM, DeAngelo DJ, Stelljes M, Martinelli G, Liedtke $\mathrm{M}$, Stock W, et al. Inotuzumab ozogamicin versus standard therapy for acute lymphoblastic leukemia. N Engl J Med. 2016;375:1-14.

12. National Cancer Institute. FDA Approves Inotuzumab for Adults with B-Cell Acute Lymphoblastic Leukemia. https://www.cance r.gov/news-events/cancer-currents-blog/2017/fda-inotuzumab -leukemia. Accessed: 11 Apr 2018.

13. U.S. Food \& Drug Administration. FDA approves inotuzumab ozogamicin for relapsed or refractory B-cell precursor ALL. https ://www.fda.gov/Drugs/InformationOnDrugs/ApprovedDrugs/ ucm572133.htm. Accessed 11 Apr 2018.

14. BESPONSA (inotuzumab ozogamicin) for injection, for intravenous use [full prescribing information]. Initial U.S. Approval: 2017. Revised: 03/2018. Pfizer, New York, NY. http://labeling. pfizer.com/ShowLabeling.aspx $?$ id=9503\&format=PDF. Accessed 1 June 2018

15. European Medicines Agency. BESPONSA Assessment Report. https://www.ema.europa.eu/documents/assessment-report/bespo nsa-epar-public-assessment-report_en.pdf. Accessed 28 Jan 2019. 2017.

16. Kantarjian H, DeAngelo D, Stelljes M, Liedtke M, Stock W, Gokbuget N, et al. Inotuzumab ozogamicin vs standard of care in patients with relapsed/refractory acute lymphoblastic leukemia: long-term results of the phase 3 INO-VATE study. American
Society of Hematology (ASH) Annual Meeting \& Exposition. 2017. p. 59.

17. Song J, Ma Q, Gao W, Cong Z, Xie J, Zimmerman Z, et al. Matching-adjusted indirect comparison of blinatumomab vs. inotuzumab ozogamicin for adults with relapsed/refractory acute lymphoblastic leukemia. Adv Ther. 2018;36:1-12.

18. Signorovitch JE, Sikirica V, Erder MH, Xie J, Lu M, Hodgkins PS, et al. Matching-adjusted indirect comparisons: a new tool for timely comparative effectiveness research. Value Health. 2012;15(6):940-7.

19. Delea TE, Amdahl J, Boyko D, Hagiwara M, Zimmerman ZF, Franklin JL, et al. Cost-effectiveness of blinatumomab versus salvage chemotherapy in relapsed or refractory Philadelphiachromosome-negative b-precursor acute lymphoblastic leukemia from a US payer perspective. J Med Econ. 2017;20:1-23.

20. Weinstein MC, O'Brien B, Hornberger J, Jackson J, Johannesson $\mathrm{M}, \mathrm{McC}$ abe $\mathrm{C}$, et al. Principles of good practice for decision analytic modeling in health-care evaluation: report of the ISPOR Task Force on Good Research Practices-Modeling Studies. Value Health. 2003;6(1):9-17.

21. US Bureau of Labor Statistics. CPI-All Urban Consumers (Current Series). Medical care in U.S. city average, all urban consumers, not seasonally adjusted. https://data.bls.gov/timeseries/ CUUR0000SAM?output_view=pct_12mths. Accessed 27 Nov 2018.

22. Guyot P, Ades AE, Ouwens MJ, Welton NJ. Enhanced secondary analysis of survival data: reconstructing the data from published Kaplan-Meier survival curves. BMC Med Res Methodol. 2012;1(12):9.

23. Schoenfeld D. Residuals for the proportional hazards regresssion model. Biometrika. 1982;69(1):239-41.

24. Phillippo DM, Ades AE, Dias S, Palmer S, Abrams KR, Welton NJ. Methods for population-adjusted indirect comparisons in health technology appraisal. Med Decis Making. 2018;38(2):200-11.

25. Amdahl J. Package 'flexsurvcure' July 17, 2017. https://cran.rproject.org/web/packages/flexsurvcure/flexsurvcure.pdf. Accessed 30 June 2018.

26. Jackson C. Package 'flexsurv'. Flexible Parametric Survival and Multi-State Models. May 11, 2016. https://cran.r-project.org/web/ packages/flexsurv/flexsurv.pdf. Accessed 30 Jan 2016.

27. Lambert PC, Thompson JR, Weston CL, Dickman PW. Estimating and modeling the cure fraction in population-based cancer survival analysis. Biostatistics. 2007;8(3):576-94.

28. Rowen D, Brazier J, Young T, Gaugris S, Craig BM, King MT, et al. Deriving a preference-based measure for cancer using the EORTC QLQ-C30. Value Health. 2011;14(5):721-31.

29. Liang KY, Zeger SL. Longitudinal data analysis using generalized linear models. Biometrika. 1986;73:13-22.

30. Sullivan PW, Ghushchyan V. Preference-Based EQ-5D index scores for chronic conditions in the United States. Med Decis Making. 2006;26(4):410-20.

31. Barlev A, Lin VW, Song X. Burden of hospitalization in relapsed acute lymphoblastic leukemia. Curr Med Res Opin. 2016;32(7):1209-12.

32. Blue Cross and Blue Shield of Michigan. Medicare Advantage PPO Enhanced Benefits. Fee Schedule. https://www.bcbsm.com/ content/dam/public/Providers/Documents/help/medicare-advan tage-fee-schedule.pdf. Accessed 27 Nov 2018.

33. Center for Medicare and Medicaid Services. Physician Fee Schedule Search. https://www.cms.gov/apps/physician-feeschedule/search/search-results. aspx $? \mathrm{Y}=0 \& \mathrm{~T}=0 \& \mathrm{HT}=0 \& \mathrm{CT}=$ $3 \& \mathrm{H} 1=96521 \& \mathrm{M}=5$. Accessed on 27 Nov 2018.

34. National Institute for Health and Care Excellence. Single Technology Appraisal. Inotuzumab ozogamicin for treating relapsed or refracotry B-cell acute lymphoblastic leukaemia [ID893]. 
Committee Papers. 2017. https://www.nice.org.uk/guidance/gidta10091/documents/committee-papers-2. Accessed 15 June 2017.

35. IBM Corporation. IBM Micromedex RED BOOK. http://www. micromedexsolutions.com/home/dispatch. Accessed 27 Nov 2018.

36. Dasta JF, McLaughlin TP, Mody SH, Piech CT. Daily cost of an intensive care unit day: the contribution of mechanical ventilation. Crit Care Med. 2005;33(6):1266-71.

37. Zhang X, Song X, Lopez-Gonzalez L, Jariwala-Parikh K, Romanov V, Cong Z. Economic Burden of veno-occlusive disease (VOD) in patients with acute lymphoblastic leukemia (ALL) in the US. Blood. 2017;130(Suppl 1):5611.

38. Zhang X, Song X, Lopez-Gonzalez L, Jariwala-Parikh K, Cong Z. Economic burden of hematopoietic stem cell transplantation (HSCT) in patients with acute lymphoblastic leukemia (ALL) in the US. Blood. 2017;130(Suppl 1):3376.

39. Sanders GD, Neumann PJ, Basu A, Brock DW, Feeny D, Krahn M, et al. Recommendations for conduct, methodological practices, and reporting of cost-effectiveness analyses: second panel on cost-effectiveness in health and medicine. JAMA. 2016;316(10):1093-103.

40. Martin PJ, Counts GW Jr, Appelbaum FR, Lee SJ, Sanders JE, Deeg HJ, et al. Life expectancy in patients surviving more than 5 years after hematopoietic cell transplantation. J Clin Oncol. 2010;28(6):1011-6.

41. Stelljes M, Su Y, Fahrbach K, Vandendries E, Page V, Onyekwere $\mathrm{U}$, et al. Indirect Treatment Comparison (ITC) of Inotuzumab Ozogamicin ( $\mathrm{InO}$ ) and Blinatumomab (Blina) for Relapsed or Refractory (RR) Acute Lymphoblastic Leukemia (ALL). Blood. 2017; 130:2558.

42. Chastek B, Harley C, Kallich J, Newcomer L, Paoli CJ, Teitelbaum AH. Health care costs for patients with cancer at the end of life. J Oncol Pract. 2012;8(6):75s-80s. 